\title{
Harnessing the Wisdom of the Confident Crowd in Medical Image Decision-making
}

\author{
Eeshan Hasan ${ }^{1}$, Quentin Eichbaum², Adam C. Seegmiller ${ }^{2}$, Charles Stratton ${ }^{2}$, Jennifer S. \\ Trueblood $^{1}$ \\ ${ }^{1}$ Department of Psychological and Brain Sciences, Indiana University \\ ${ }^{2}$ Department of Pathology, Microbiology, and Immunology, Vanderbilt University Medical \\ Center
}




\section{Author Note}

\section{Corresponding Author:}

Jennifer Trueblood

Department of Psychological and Brain Sciences, Indiana University

1101, E. 10th St. Bloomington, IN, USA 47405-7007

email: jstruebl@iu.edu

CRediT Statement Eeshan Hasan: Conceptualization, Methodology, Data curation Formal analysis, Software, Visualization, Writing - Original Draft;

Quentin Eichbaum: Conceptualization, Resources, Writing - Review \& Editing, Funding Acquisition;

Adam Seegmiller: Conceptualization, Resources, Writing - Review \& Editing, Funding Acquisition;

Charles Stratton: Conceptualization, Resources, Writing - Review \& Editing, Funding Acquisition;

Jennifer Trueblood: Conceptualization, Investigation, Methodology, Writing - Original Draft, Supervision, Project Administration, Funding Acquisition

Acknowledgements This work was supported by a Clinical and Translational Research Enhancement Award from the Department of Pathology, Microbiology, and Immunology, Vanderbilt University Medical Center. This work was also supported by NSF grant 1846764 . We thank Payton O’Daniels for his excellent research assistance.

Data Availability Data for all experiments is available on the Open Science Framework at https://osf.io/ckvxz/. 


\begin{abstract}
Improving the accuracy of medical image interpretation is critical to improving the diagnosis of many diseases. Using both novices (undergraduates) and experts (medical professionals), we investigated methods for improving the accuracy of a single decision maker and a group of decision makers by aggregating repeated decisions in different ways. Participants made classification decisions (cancerous versus non-cancerous) and confidence judgments on a series of cell images, viewing and classifying each image twice. We first examined whether it is possible to improve individual-level performance by using the maximum confidence slating algorithm (Koriat, 2012b), which leverages metacognitive ability by using the most confident response for an image as the 'final response'. We find maximum confidence slating improves individual classification accuracy for both novices and experts. Building on these results, we show that aggregation algorithms based on confidence weighting scale to larger groups of participants, dramatically improving diagnostic accuracy, with the performance of groups of novices reaching that of individual experts. In sum, we find that repeated decision making and confidence weighting can be a valuable way to improve accuracy in medical image decision-making and that these techniques can be used in conjunction with each other.

Keywords: Wisdom of the Crowds; Metacognition; Confidence; Experts; Pathology image interpretation
\end{abstract}




\section{Harnessing the Wisdom of the Confident Crowd in Medical Image Decision-making}

The diagnosis of several diseases and underlying conditions is dependent on interpretations and diagnostic decisions made by medical professionals after viewing medical images. Despite specialized training and advancement in technology, diagnostic mistakes still occur. One successful approach to reducing diagnostic errors is to use second opinions. For example, the misclassification rate decreased from $24.7 \%$ to $18.1 \%$ in breast histopathology with multiple readings (Elmore et al., 2016). In parallel, in the decision making literature, in a phenomenon called the Wisdom of the Crowds (WoC), it has been shown that aggregating responses of several individuals can lead to an improvement in overall accuracy (Galton, 1907; Surowiecki, 2005). Importantly, studies have shown that WoC generalizes to medical image decision making (Juni \& Eckstein, 2017; Kurvers et al., 2016; Wolf et al., 2015). Additionally, there has been recent interest in the relationship between accuracy and confidence in expert medical image decision making (Litvinova et al., 2022). In this paper, we build on both lines of inquiry and examine whether incorporating confidence judgments into decision aggregation algorithms improves accuracy in medical image diagnosis for both novices and experts.

Despite evidence demonstrating that second opinions can reduce errors, multiple readings are not always performed. In the United States this is because it is considered to be time-consuming and the additional readings are not always reimbursed (Waite et al., 2017). In other parts of the world such as Africa, due to a lack of trained pathologists (Nelson et al., 2018), multiple readings are difficult to obtain. Intriguingly, in the decision making literature, decision makers might be able to act as their own fresh pair of eyes by repeatedly making the same decision. In a phenomenon called the Wisdom of the Inner Crowd (WoIC), it has been shown that aggregating multiple decisions from the same individual on the same stimulus can boost accuracy (Herzog \& Hertwig, 2009, 2014; Koriat, 2012b; Litvinova et al., 2020; Litvinova et al., 2022; Stroop, 1932; Vul \& Pashler, 2008). However, most of these studies have used simple perceptual stimuli and general knowledge questions. Only one of these studies has examined WoIC in complex perceptual tasks, such as medical image decision making (Litvinova et al., 2022). 
In our experiments, participants made decisions about images of white blood cells. The classification of these images into blast (cancerous) and non-blast (non-cancerous) forms a crucial step in the identification of lymphoma or leukemia (Trueblood et al., 2018). In a set of experiments, participants (both experts and novices) were asked to classify these images as blast or non-blast cells. For each image, we collected two classification decisions along with their corresponding confidence judgment from every individual. We applied WoIC and WoC algorithms by leveraging information obtained through their confidence judgments to combine decisions. First, we systematically tested whether confidence information can be used to improve the decisions made by a single participant and how this compares to using different participants. Further, we were interested in testing whether improvements made at the individual level, as shown in Litvinova et al. (2022), scale to the group level, where decisions from multiple individuals are combined. We also examined different ways of combining confidence decisions. For example, with two decisions and confidence judgments on a given image, one could take the more confident response or the average decision of the two responses. Finally, we tested the set of aggregation algorithms for both novice and experts. Thus, the work presented here represents the first comprehensive evaluation of wisdom of the crowd methods for medical image decision-making, taking into consideration both individual level and group level aggregation methods, confidence, and expertise.

WoC algorithms have been robustly applied in several domains including politics, Google searches, and financial markets (Surowiecki, 2005). Diversity or variation of responses has been identified as crucial to the successful application of WoC (Davis-Stober et al., 2014; Surowiecki, 2005). For WoC, this amounts to having a diverse set of participants, independence of decision makers, and having private judgments (Surowiecki, 2005). Importantly, an increase in diversity must be meaningful. For example, one might add diversity by adding participants with random decisions which will just add noise to the system. In this case, increasing diversity will not improve WoC.

Since several of the conditions for increasing diversity in WoC are inapplicable to WoIC, 
researchers have found creative ways of eliciting a diverse or independent set of responses from the same individual. One such technique called dialectic bootstrapping, involves eliciting two decisions based on opposing sets of information, such that the biases present in both decisions cancel out. This is practically implemented by re-framing the question to make the participant consider the opposite perspective (Herzog \& Hertwig, 2009, 2014; Montgomery \& Lee, 2022). In medical image decision making, one might re-frame the question - 'Is this a blast cell?' to the opposite - 'Is this a non-blast cell?'. Another implementation of dialectic bootstrapping in medical image decision making could be to slightly change the decision-maker's perspective by rotating the image ${ }^{1}$ In our experiments, we leveraged both methods in order to increase the diversity of responses within an individual.

Metacognitive abilities allow people to track their mistakes and make confidence judgments that often correlate with objective performance (Fleming et al., 2012; Griffin \& Tversky, 1992; Yeung \& Summerfield, 2012). This information can be used to weight decisions during the aggregation process. In the context of WoIC, this can be used to increase the variation in responses, particularly for binary choice tasks (e.g., deciding cancerous versus non-cancerous) by adding a continuous scale on which the decision maker can vary their response. Thereby, we effectively convert a discrete decision problem in which a participant can choose between two options, to a problem with a continuous outcome. However, the use of confidence judgments has limitations. For example, self-reported confidence judgments are known to have large individual differences (J. Baron et al., 2014; Pallier et al., 2002). Second, self-reported confidence is a noisy measure that is often not well calibrated (Attali et al., 2020; Griffin \& Brenner, 2004; Griffin \& Tversky, 1992; Olsson, 2014; Rahnev \& Fleming, 2019; Shekhar \& Rahnev, 2021). This leads to patterns of overconfidence and underconfidence that are thought to interact with factors such as difficulty. For example, the overconfidence bias has been documented in medical image decision-making (Saposnik et al., 2016).

Despite these shortcomings, the 'maximum confidence slating algorithm' has been

\footnotetext{
${ }^{1}$ Images of white blood cells do not have a natural orientation, unlike other medical images such as lung x-rays.
} 
successfully applied to perceptual decision-making tasks with binary categorization responses (Koriat, 2012b). In a pair of decision makers, or a 'dyad', the algorithm considers the more confident response as the final response. For example, in Koriat (2012b) two participants were presented two shapes in a two alternative forced choice task and were asked to indicate which one they thought was longer and to provide their confidence in their answer. After normalizing the confidence ratings (Koriat, 2012a), the more confident response was considered to be the final response of the dyad. This final response was shown to be more accurate than the individual responses. In Koriat (2012b), this result generalized to the case where both of the responses in the 'dyad' were from the same decision maker, albeit with a smaller improvement. Litvinova et al. (2022) showed that this algorithm, using the same person, extends to expert medical decision making as well. In this paper, we tested both versions of the algorithm - with the same and different decision makers forming the dyad.

Recently, there has been interest in crowd sourcing a large number of novice decisions for medical diagnosis or to create labeled data sets to train artificial intelligence systems (Alialy et al., 2018; Dissanayake et al., 2019; Kamar et al., 2012; Mozafari et al., 2014; Ørting et al., 2020). However, previous research by Kurvers et al. (2016), Litvinova et al. (2022), and Wolf et al. (2015) has only examined data from expert participants. Hence, WoC for novice participants remains to be demonstrated. The idea of using a distributed network of individuals to make diagnostic decisions has attracted the attention of investors and led to the development of companies to classify medical images (Press, 2021). One such company uses an app based set-up where individuals with differing amounts of expertise (e.g., individuals with no medical training, medical students, residents, and attending physicians) are used to label medical images. In such a set-up, one might be able to collect multiple responses and their confidence rating on some images with relative ease.

For binary decision tasks, such as the one we investigate in this paper, previous research has shown that the relationship between group size and crowd accuracy is domain specific (Lee \& Lee, 2017). In some cases, having a larger group size does not lead to improved accuracy when 
the majority response is used as the group decision. In a separate line of research, it has been shown that confidence weighting is an effective method for combining the decisions of a group of people performing simple laboratory tasks (Meyen et al., 2021). Here we extend this idea to the real-world problem of medical image decision-making and explore a wider set of confidence based aggregation algorithms. For a dyad, in the absence of additional information, confidence slating is the only way to break an inconsistent set of responses. With more than two participants, there are more ways of resolving inconsistencies. For example, we might be able to 'weight' decisions such that one confident decision is weighted more heavily than two decisions that are not confident. To this end, we designed different WoC algorithms. First, we compared algorithms based on simple voting and compared them to algorithms based on votes that were weighted by confidence judgments. We weight confidence judgments either by the confidence values provided or by the log odds transformation of the confidence values. Second, we tested the value of using repeated decisions from the same decision maker and hence compared algorithms based on two repeated decisions to algorithms based only on one decision per person. Finally, we compared algorithms where we slated the decision to the more confident response to the algorithms based on confidence weighting.

As mentioned above, all the experiments were based on classification decisions made on images of white blood cells. We conducted two experiments (Experiments 1a \& 1b) on novice undergraduate participants. In Experiment 1a, we used a consider the opposite technique (i.e., dialectic bootstrapping) in addition to rotating the image in the second presentation with the aim of increasing the diversity or independence of responses. In Experiment 1b, we collected data without these manipulations. For Experiment 2, we followed a procedure similar to Experiment 1a, but we used expert participants. We compared the results from Experiment 1a to Experiment $1 \mathrm{~b}$ to test the importance of the diversity manipulations. We compared the results from Experiment 1a and Experiment 2 to compare novice and expert participants. We used both novice participants and medical experts for three important reasons. First, data from novice participants provides a baseline for comparing expert participants. Second, novices and experts might be 
using different cognitive process, hence it is not clear whether our findings will generalize to expert participants. Third, there is recent interest in using novices to assist with medical image diagnosis. As mentioned above, this opens up the possibility of crowdsourcing large numbers of untrained individuals to perform simple diagnostic tasks (Alialy et al., 2018; Dissanayake et al., 2019; Ørting et al., 2020).

Data for all experiments is available on the Open Science Framework at https://osf.io/ckvxz/, (Hasan et al., 2021).

\section{Experiments 1a \& 1b: Novice Participants}

Experiment 1a and $1 \mathrm{~b}$ tested novice participants. Experiment 1a included manipulations to increase the diversity of responses as compared to Experiment $1 \mathrm{~b}$.

\section{Method}

\section{Participants}

A total of 87 undergraduate students at Vanderbilt University participated in Experiments 1a and 1b. We tested 45 participants in Experiment 1a and 42 in Experiment 1b. The sample size was based on similar studies examining pathology image based medical decision making (Trueblood et al., 2018). The participants primarily identified as female (Exp. 1a: 76\%; Exp. 1b: $70 \%)$. The mean age was 18.9 years $(\mathrm{SD}=1.2$; IQR $18-24)$ for Experiment 1a and 19.5 years $(\mathrm{SD}=2.5$; IQR-18 - 20) for Experiment 1b. Participants received course credit for participating. We refer to these as the novice participants.

\section{Materials}

The set of stimuli were identical to Trueblood et al. (2018), consisting of 300 digital images of Wright-stained white blood cells taken from anonymized patient peripheral blood smears at Vanderbilt University Medical Center (VUMC). The images were taken by the CellaVision DM96 (CellaVision AB, Lund, Sweden). Examples of such an images can be seen in Figure 1. Half (150) of these images were blasts (cancerous) and the other half were non blasts 
(non-cancerous). Within each category, half (75) were classified as easy and the other half were classified as hard. These labels were based on consensual judgments from three hematopathologists from the Department of Pathology at Vanderbilt University Medical Center. Further details on image curation can be found in Trueblood et al. (2018). All of these images were used in Experiment 1a and 1b. The experimental protocol was approved by the Institutional Review Board at Vanderbilt University.

\section{Procedure}

The experiments were designed to collect two classification responses and their corresponding confidence judgments on each image. The general overview of the experimental design can be seen in Figure 1. Participants first completed a familiarization block, training block, and practice trials before starting the main task. The four cell types (blast vs non-blast $\mathrm{x}$ easy vs hard) were counterbalanced in each of these initial blocks. In the 36 familiarization trials, participants viewed cell images with their corresponding labels (either blast or non-blast) one at a time for as long as they wanted. In the 60 training trials, participants viewed two cell images and their task was to select the image that matched a label (either blast or non-blast) at the top of the screen. They received feedback in these training trials. Finally, in the 20 practice trials, they were shown a single cell image and instructed to indicate whether the cell was a blast cell or a non-blast cell and received feedback.

The main task consisted of two parts, each with 300 trials corresponding to the 300 unique images contained in the stimuli set. Across the two parts of the main task, participants saw a total of 600 images, so that each image was shown twice. On each trial, participants were shown a single image and had to decide if it was a blast or non-blast cell. In addition to making a choice

on each trial, participants were also instructed to report how confident they were that they selected the correct response on a scale ranging from 50\% (guessing) to 100\% (certain correct).

In Experiment 1a, they were asked "Is this a blast cell?" for the first part of the main trials but were asked "Is this a non-blast cell?" for the second part. In Experiment 1b, the second block 
was the same as the first (i.e., they were asked "Is this a blast cell?") for the entire main task. In addition, the images in the second half of the main task of Experiment 1a were rotated 180 degrees while there was no rotation for Experiment 1b. Experiment 1a had 20 practice trials with feedback in between both parts of the main trials to help with the transition in instructions.

\section{Behavioral Results}

In this section, we present the behavioral results, focusing on intra-rater disagreement and the relationship between cell type, confidence and accuracy. WoIC and WoC results are presented in separate sections after Experiment 2.

Participants were excluded if their accuracy on the practice trials was less than or equal to $50 \%$. This is because these participants either did not understand the task or were not interested. We excluded 8 participants from Experiment 1a and 10 participants for Experiment $1 \mathrm{~b}$ for this reason. Two participants in Experiment 1a gave 64 and 219 responses respectively outside the valid confidence range of 50-100. Other participants in Experiment 1a had fewer than 20 responses outside the valid range. Hence, we excluded those two participants from Experiment 1a. No participants gave responses outside the valid confidence range for Experiment $1 \mathrm{~b}$. We also removed 1 participant in Experiment 1a and 1 participant from Experiment $1 \mathrm{~b}$ that gave the same response for more than $95 \%$ of the trials in either part of the main task. We removed these additional participants because we did not want to bias our analysis that examines blast and non-blast cell images separately. We ran the analysis for the main results in the paper without these exclusions and present those in the supplementary materials. The results remain qualitatively the same. After these exclusions, we retained 34 out of 45 participants in Experiment 1a and 31 out of 42 participants in Experiment 1b. For Experiments 1a and 1b, the mean accuracy was $66.1 \%(\mathrm{SD}=8.8$, IQR $60.1 \%-71.5 \%)$ and $66.5 \%(\mathrm{SD}=10.7$, IQR $59.5 \%-74.8 \%)$, respectively. These were both significantly different from chance (Experiment 1a: $t(33)=10.70$, $p<0.0001$; Experiment 1b: $t(30)=9.09, p<0.0001)$.

Since we were interested in understanding the effectiveness of the algorithms for blast and 
non-blast images separately, we also report the sensitivity (the accuracy on images that are true blasts) and the specificity (the accuracy on images that are true non-blasts) in Table 1. For Experiment 1a, the sensitivity was $63.2 \%$ and specificity was $69.0 \%$. For Experiment $1 \mathrm{~b}$, the sensitivity was $64.2 \%$ and specificity was $68.9 \%$. To test whether these differences were significant, we conducted a 2 (classification: blast, non-blast) repeated measures ANOVA with accuracy as the dependent variable. For Experiment 1a, we observed a main effect of classification, $F(1,33)=6.53, p=0.0154$. For Experiment $1 \mathrm{~b}$, the main effect of classification was not significant, $F(1,30)=1.78, p=0.1927$. Taken together, these results suggest that participants are better at detecting non-blast cells in Experiment 1a.

Since one of our main goals with Experiments 1a and $1 \mathrm{~b}$ was to investigate methods for increasing the diversity of responses within individuals, we calculated and compared the intra-rater disagreement for each participant. We report the results in Table 1. Since participants made two responses on each image, we calculated the rate at which both of these responses were different. The intra-rater disagreement rate was significantly higher $(t(63)=2.34, p=0.022)$ in Experiment 1a (30.2\%) than Experiment $1 \mathrm{~b}(23.5 \%)$. We conducted a 2 way repeated measures ANOVA with inconsistency as the dependent variable and classification as the independent variable. For both the experiments, the inconsistency rate was higher for blasts than for non-blasts (Exp. 1a: blast: $33.1 \%$ non-blast: $27.4 \% ; F(1,33)=9.16, p=0.0048$; Exp 1b: blast: $25.8 \%$ non-blast: $21.2 \% ; F(1,30)=11.72, p=0.0018)$. This shows that the manipulations used in Experiment 1a to increased the diversity of responses as intended

Next, we compared the diversity obtained when we considered two responses from the same person to the diversity obtained when we considered two responses from two different people. To this end, we calculated the inter-rater disagreement by pairing the participants randomly and calculating the inter-rater disagreement with their first responses. We repeated this process and report the mean inter-rater disagreement. Since both the experiments were identical for the first set of main trials, we do not expect a difference in the inter-rater disagreement in Experiment 1a and Experiment 1b. For Experiment 1a, the inter-rater disagreement was $41.1 \%$ 
and for Experiment 1b, the inter-rater disagreement was $40.9 \%$. These were not significantly different $(t(63)=0.04, p=0.9704)$. However, this did not exceed the diversity gained by using another person.

We normalized the confidence ratings before using them due to differences in the participants' self-reported confidence. Additionally, as participants gained experience with the task, it is possible that they changed the way that they used the confidence scale over the course of the experiment. Further, in Experiment 1a, the instructions changed between the two parts of the main task. To determine if confidence ratings for the two parts of the main task came from the same distribution, we conducted a Kolmogorov-Smirnov (KS) test. We observed that for 18 out of 34 participants in Experiment 1a and 18 out of 31 participants in Experiment 1b, the distribution of confidence ratings in the two parts of the main task were significantly different $(p<0.05)$. Hence, we normalized the confidence ratings for the two parts of the main task separately. In other words, we calculated the z-score of the confidence ratings for each person separately for each part of the main task. In this paper, we examine algorithms with and without this normalization step.

We next examined how confidence was related to the accuracy, image type, and difficulty across all trials in the main task (see Figure 2 panels A and B). We conducted a 2 (accuracy: correct, incorrect) x 2 (classification: blast, non-blast) x 2 (difficulty: easy, hard) repeated measures ANOVA with normalized confidence as the dependent variable. We observed a significant main effect for accuracy (Exp. 1a: $F(1,33)=97.9 p<0.0001$; Exp. 1b: $F(1,30)=71.8, p<0.0001)$, where the confidence was higher for accurate responses. We also observe a main effect of classification (Exp. 1a: $F(1,33)=34.7, p<0.0001$; Exp. 1b: $F(1,30)=19.5, p=0.0001)$, where participants were more confident on non-blast images than blast images. We also found a main effect of difficulty in $\operatorname{Exp} .1 \mathrm{a}(F(1,33)=7.2, p=0.0114)$, where participants were more confident on easy images. The main effect of difficulty was not significant for Exp. $1 \mathrm{~b}(F(1,30)=2.3, p=0.1407)$. We also found significant interactions between classification and difficulty in both experiments, showing the difference in confidence for 
blast and non-blast images was larger for easy images as compared to hard images. Importantly, participants gave higher confidence ratings when they were accurate, showing that confidence reflects accuracy, which is critical for confidence-based aggregation algorithms.

We calculated the Brier score and Murphy's Brier decomposition for each experiment (Yates, 1982). The Brier score is the mean squared error between the confidence rating and the outcome. The Brier score decomposes into resolution and reliability scores. The resolution is a measure of the extent to which individuals can distinguish between blast and non-blast cells. The reliability score is a measure of how well these confidence judgments are calibrated towards the probability of the outcomes. We briefly describe how these measures are calculated. First, the base rate $(r)$ is calculated for the outcome. To calculate the resolution and reliability score, the confidence judgments are segregated into $k$ equally sized bins. For each bin $(b)$, we calculate the mean confidence judgment $\left(\bar{C}_{b}\right)$, the number of judgments given $\left(n_{b}\right)$, and the rate at which the associated outcome $\left(\bar{X}_{b}\right)$ occurs in that bin. The resolution is then calculated as the mean squared deviation between the base rate and the rate at which the associated outcome takes place in each bin, weighted by the number of confidence judgments in that bin, which is given by the formula $\sum_{b} n_{b}\left(\bar{X}_{b}-r\right)^{2} / n$. Hence, larger values of resolution indicate an ability to distinguish between blast and non-blast cells by measuring their deviation from the base rate for each bin. The reliability score is the mean squared deviation between the mean confidence judgment and the realized rate at which the outcomes occur in a given bin weighted by the number of decisions made. This is given by the formula $\sum_{b} n_{b}\left(\bar{C}_{b}-\bar{X}_{b}\right)^{2} / n$. Lower values of the reliability score indicate that the judge gives reliable judgments.

For our calculations, the outcome was the cell being cancerous. For the WoC algorithms presented later, it is important for the confidence judgments to have the same scale. To this end, we pooled all of the confidence judgments from all individuals together and calculated the mean and standard deviation of the pooled confidence judgments. We then re-scaled the z-scores of individual participants to the population's mean and standard deviation. The Brier score results that we mention in the paper are qualitatively the same even without this normalization. Hence, 
we present the results with normalized confidence because we use those values in the algorithms we examine in the paper. We converted the original half range confidence scale from $50-100$ scale to a full range confidence scale from $0-100$ ) scale by flipping the non-cancerous judgments such that 0 was where the individual confidently decided that the cell was not cancerous and 100 was where the individual was confident that the cell was cancerous. We binned the confidence judgments into 10 bins $(0-10,10-20, \ldots, 90-100)$.

Since the focus of this paper is to examine the best way of combining confidence judgments, we also calculate the Brier score decomposition for the two different ways in which confidence judgments can be combined when there are repeated decisions on images. The first method, called maximum confidence (MC) uses the more confident judgment as the final aggregated judgment. Alternatively, one could use the average confidence of the two responses (AC). For Experiment 1a, AC had a significantly higher resolution (MC: 0.062 AC: 0.067, $t(33)=3.64 ; p=0.0003)$. AC had a significantly lower reliability score (MC: 0.058 AC: 0.075, $t(33)=5.47 ; p<0.0001)$. AC had a significantly lower Brier score (MC: 0.249 AC: 0.221, $t(33)=6.64 ; p<0.0001)$. The results are similar for Experiment $1 \mathrm{~b}$. AC had a significantly higher resolution (MC: $0.056 \mathrm{AC}: 0.060, t(30)=5.04 ; p<0.0001)$. AC had a significantly lower reliability score $(\mathrm{MC}: 0.059 \mathrm{AC}: 0.030, t(30)=6.12 ; p<0.0001)$. AC had a significantly lower Brier score (MC: 0.257 AC: 0.221, $t(30)=6.64 ; p<0.0001)$. Hence, we observe that averaging the confidence judgments creates final judgments that are better able to distinguish between blast and non-blast cells while being more reliable and accurate judgments compared to using the more confident judgment.

\section{Discussion}

Experiments 1a and 1b were designed to collect data so that we could apply our WoC and WoIC algorithms to it. We first observed that the novice participants learned to do the task with the brief training that they received. Their performance was similar for both the cell types with a slightly higher accuracy for non-blasts. Second, we also observed that the manipulations of 
dialectic bootstrapping and rotation increased the intra-rater variance. It is difficult to know the separate impact of the two manipulations. They could have had independent effects or have interacted to bring about this difference. However, as shown in the Supplement, the change in response rate for blast cells over the two main blocks in Experiment 1a is unlikely to be explained by the rotation manipulation alone. This is because the rotation manipulation would probably affect blast and non-blast cells equally and not cause a change in the response rate. Third, we observed that the increase in intra-rater variance did not exceed that of using two different decision makers. Fourth, we also observed that confidence was related to accuracy such that more confident responses were the ones that were also accurate. This shows that the confidence data that we collected was meaningful and that it might be usable for aggregation algorithms, which are described in later sections. Finally, the comparison between $\mathrm{MC}$ and $\mathrm{AC}$ suggests that averaging confidence gives us responses that are better able to distinguish blast and non-blast cells while being more reliable and better at predicting the outcome.

\section{Experiment 2: Expert Participants}

This experiment is similar to Experiment 1a except that it uses expert participants instead of novice participants.

\section{Method}

\section{Participants}

A total of 23 pathologists and laboratory professionals participated in Experiment 2. Participants were recruited at the American Society for Clinical Pathology (ASCP) annual conference held in Baltimore, Maryland, USA in October 2018. The participants primarily identified as female (73\%). The mean age was 42.4 years ( $\mathrm{SD}=13.5$; IQR $30-56)$. Participants were compensated with a $\$ 10$ Starbucks gift card for participating. The sample size was of convenience. We refer to these as the expert participants. The experimental protocol was approved by the Institutional Review Board at Vanderbilt University. 


\section{Materials}

A subset of 60 out of the 300 images that were used in Experiment 1 were used in Experiment 2. All the images were classified as hard. The images were counterbalanced across the two categories - blast and non-blast.

\section{Procedure}

Similar to Experiment 1, this experiment was designed to collect two classification responses and their corresponding confidence judgments on each image. It was shorter due to time constraints at the ASCP conference. So that the task was not too easy, expert decisions were only collected for the hard cell types. Since the experts were experienced with these kinds of cells, the training phase was also shortened. It consisted of 20 trials of hard cells counterbalanced among blast and non-blast, where they had to match the cell to its corresponding label. They received feedback in these trials. Following the training block, experts did 20 practice trials with easy cells counterbalanced across blast and non-blast to become familiar with the interface for the main trials.

The main task consisted of two parts, each containing 60 images. Similar to Experiment 1a, both the main blocks had the same images. After each decision, expert participants were also asked to indicate their confidence in their decision. They did not receive feedback in these trials. Similar to Experiment 1a, there was a change in instruction ('Is this a blast cell?' to 'Is this a non-blast cell?') between the two parts of the main task and the images were rotated 180 degrees in the second half of the main trials.

\section{Behavioral Results}

For Experiment 2, 1 out of the 23 experts was excluded because they did not provide any confidence ratings on the first part of the main task. None of the participants had an accuracy less than or equal to $50 \%$. The mean accuracy of the experts was $71.6 \%(\mathrm{SD}=14.3$; IQR $60.1-83.9)$, which was significantly above chance $(t(21)=6.922, p<0.0001)$. Since the stimuli were 
different for novices and experts, we also report the novice accuracy on the subset of stimuli seen by experts in Experiment 2. On this reduced set of images, the mean accuracy was $61.8 \%$ $(\mathrm{SD}=10.7$; IQR 53.3\% - 69.3\%) for Experiment 1a and 59.0\% (SD=9.8; IQR 51.3\% - 63.3\%) for Experiment 1b, both were significantly above chance (Experiment 1a: $t(33)=6.44$, $p<0.0001$; Experiment 1b: $t(30)=5.11, p<0.0001)$. As expected, experts performed better than novices $(t(54)=6.922, p<0.0001)$.

Once again, we break down the accuracy separately for the blast and non-blast images. The sensitivity was $71.0 \%$ and specificity was $72.2 \%$. The average rate of responding blast was 49.4\%. This was not significantly different from $50 \%(t(21)=0.27, p=0.793)$. However, the blast response rate changed significantly from $46.7 \% \%$ to $52.0 \%$ over the two blocks in the main block $(t(21)=2.35, p=0.0288)$.

As for intra-rater disagreement, we restricted the responses made by participants in Experiment 1a to the stimuli used in Experiment 2 and compared the intra-rater disagreement. The experts in Experiment 2 had a lower intra-rater disagreement $(22.3 \%)$ than novices in Experiment 1a (31.9\%). This shows that experts had more consistent responses than novices. We conducted a repeated measures ANOVA with classification as the independent variable and inconsistency as the dependent variable to test if experts were more consistent on one of the cell types. There was no significant difference in the inconsistency rate between the blast and non-blast cells (blast 22.9\%, non-blast $21.5 \% ; F(1,21)=0.26, p=0.6171$ ).

With the same procedure as in Experiment 1, we calculated the mean inter-rater disagreement. The mean inter-rater disagreement for experts is $40.7 \%$. This shows that like the novices, combining different experts provides more diverse responses than repeatedly asking the same expert the same question.

As discussed above, we conducted the Kolmogorov Smirnoff test to determine whether confidence ratings should be normalized differently for the two main blocks. For 6 out of 22 participants in Experiment 2, the confidence judgments came from different distributions $(\mathrm{p}<0.05)$. Hence, we normalized the confidence by calculating the $\mathrm{z}$-score separately for the two 
main blocks as discussed above. When combining the decisions from multiple individuals, it was important for the confidence judgments to be on the same scale. Hence, we re-scaled the confidence judgments by centering them at the population mean and standard deviation.

We examined how confidence was related to accuracy, image type, and difficulty across all trials in the main task. These relationships can be seen in Figure 3 , We conducted a 2 (accuracy: correct, incorrect) x 2 (classification: blast, nonblast) repeated measures ANOVA for experts. Similar to the novices, we observed a significant main effect for accuracy $(F(1,21)=36.6$, $p<0.0001)$, where participants were more confident on trials where they were accurate. There was no main effect of classification for experts $(F(1,21)=0.0, p=0.9062)$. This shows once again that confidence is positively correlated with accuracy.

As in Experiment 1, we compared the two different ways of combining the responses. That is, we compared the difference in the Brier scores between using the more confident response to averaging the confidence response. We observe that $\mathrm{AC}$ has a significantly higher resolution (MC: 0.103 . AC: $0.119 t(21)=4.42 ; p=0.0002)$. AC has a significantly lower reliability score (MC: 0.089 AC: $0.065 t(21)=2.95 ; p=0.0077)$. AC has a significantly lower Brier score (MC: 0.235 AC: $0.195 t(21)=5.14 ; p<0.0001)$. These results show that similar to Experiment $1, \mathrm{AC}$ gives us a higher resolution along with better reliability score and a better overall Brier score.

\section{Discussion}

Experiment 2 examined expert performance on the white blood cell task. First, we observed that the performance of the experts is much better than chance. This shows that despite the reduction in training, experts did the task well and are much better than the novice participants. Second, the change in instructions in the two blocks of the main trials changed the rate at which participants responded blast vs non-blast. Third, we observed that the intra-rater disagreement in the experts is lower than that of the novices. However, the disagreement rate is still substantial. Thus, a correct resolution of inconsistent responses can potentially increase accuracy appreciably. Fourth, the rate at which inconsistencies occur is similar for blast and 
non-blast cells. Fifth, the inter-rater disagreement with the expert participants is quite high. This large difference in intra-rater and inter-rater disagreement shows that combining two different decision makers might be more beneficial for expert participants. Finally, experts' confidence ratings were related to their accuracy, with correct responses being more confident than incorrect responses.

The results for the experts' Brier decomposition parallel the results for the novices. Once again, AC gives a higher resolution along with better reliability score and Brier scores. Hence, we think that it might be better to average responses than to take the more confident one.

\section{Table 1}

Accuracy, intra-rater disagreement, and performance of the maximum confidence slating algorithm for Experiments $1 a, 1 b$, and 2.

\begin{tabular}{|c|c|c|c|c|c|c|}
\hline Exp. & $\begin{array}{l}\text { Classif- } \\
\text { ication }\end{array}$ & $\begin{array}{l}\text { Average } \\
\text { Accuracy }\end{array}$ & $\begin{array}{c}\text { Intra- } \\
\text { rater } \\
\text { disagree- } \\
\text { ment }\end{array}$ & $\begin{array}{c}\text { Percentage } \\
\text { Inconsistent } \\
\text { Slated } \\
\text { Correctly }\end{array}$ & $\begin{array}{c}\text { Max } \\
\text { Confidence } \\
\text { Slating } \\
\text { Acc. }\end{array}$ & $\begin{array}{c}\text { Improvement } \\
\text { from } \\
\text { Average } \\
\text { Accuracy }\end{array}$ \\
\hline \multirow{3}{*}{$\begin{array}{c}\text { Exp. } \\
\text { 1a }\end{array}$} & Blast & $63.2 \%$ & $33.1 \%$ & $53.4 \%$ & $64.3 \%$ & $1.1 \%$ \\
\hline & Non Blast & $68.8 \%$ & $27.4 \%$ & $55.8 \%$ & $70.4 \%$ & $1.6 \%$ \\
\hline & Both & $66.0 \%$ & $30.2 \%$ & $54.5 \%$ & $67.4 \%$ & $1.4 \%$ \\
\hline \multirow{3}{*}{$\begin{array}{c}\text { Exp. } \\
\text { 1b }\end{array}$} & Blast & $64.2 \%$ & $25.8 \%$ & $53.5 \%$ & $65.1 \%$ & $0.9 \%$ \\
\hline & Non Blast & $68.9 \%$ & $21.2 \%$ & $54.0 \%$ & $69.7 \%$ & $0.8 \%$ \\
\hline & Both & $66.5 \%$ & $23.5 \%$ & $53.7 \%$ & $67.4 \%$ & $0.9 \%$ \\
\hline \multirow{3}{*}{$\begin{array}{c}\text { Exp. } \\
2\end{array}$} & Blast & $71.0 \%$ & $22.9 \%$ & $57.7 \%$ & $72.8 \%$ & $1.8 \%$ \\
\hline & Non Blast & $72.2 \%$ & $21.5 \%$ & $61.7 \%$ & $74.7 \%$ & $2.5 \%$ \\
\hline & Both & $71.6 \%$ & $22.2 \%$ & $59.6 \%$ & $73.7 \%$ & $2.1 \%$ \\
\hline
\end{tabular}

Note. This table explains the improvement obtained by maximum confidence slating with normalized confidence across the different cell types in all three experiments. The first column is the average accuracy in each experiment for each cell type. The second column looks at the intra-rater disagreement or the rate at which the two responses made on the same image in the main trials are inconsistent. The third column is the percentage or rate at which these inconsistencies are correctly resolved using the maximum confidence slating algorithm. The fourth and fifth columns contain the accuracy of the maximum confidence slating algorithm and the corresponding improvement compared to average accuracy.

\section{Wisdom of the Inner Crowd Algorithms}

In this section, we used the maximum confidence slating (MCS) algorithm, following the procedure laid out in Koriat (2012b), to aggregate individual level decisions. The MCS algorithm uses the two classification decisions for each image along with the two confidence ratings for the 
image. First, we normalized the confidence ratings as described in the behavioral results sections above. ${ }^{2}$ For each image, we used the more confident classification as the final response on that image. In this paper, we used both the responses made by the same individual for the MCS-Within algorithm. We also compared this to the results obtained when we used the first response made by two different individuals on the same image (MCS-Between).

\section{Results}

We followed the procedure of Koriat (2012) to apply the maximum confidence slating algorithm to the two responses made by the same participant. We conducted t-tests to compare the accuracy obtained by using MCS to the average performance. Calculating the average performance for the images amounts to assigning 0 to images with two incorrect responses, 1 to images with two correct responses, and 0.5 to images with one correct and one incorrect response and then calculating the average score across all images. As stated in Tables 1 and 2 , the accuracy significantly increased by $1.3 \%$ to $67.4 \%$ by resolving $54.5 \%$ of the inconsistencies in the correct direction for Experiment 1a $(t(33)=4.0, p=0.0004)$ and by $0.9 \%$ to $67.4 \%(t(30)=2.8$, $p=0.0078$ ) by resolving $53.7 \%$ of the inconsistencies in the correct direction for Experiment 1b.) As shown in Table 2, we observed that even without the normalization step for Experiment 1a and 1b, MCS improved performance (Exp. 1a: MCS - 67.6\%, $t(33)=4.06, p=0.0003$; Exp. 1b: $\operatorname{MCS}-67.3 \% \mathrm{t}(30)=2.77, \mathrm{p}=0.0095)$.

We wanted to understand whether the improvement depended on cell type. As mentioned above, the rate at which the inconsistencies occurred were slightly different for blast and non-blast cells. We calculated the rate at which MCS resolved the inconsistencies for both blast and non-blast cells towards the correct answer for both of the experiments. The rate at which the inconsistencies were resolved correctly for blast and non-blast cells was $53.4 \%$ and $55.8 \%$ respectively for Experiment 1a, leading to a reduction of false alarms by $1.1 \%$ and misses by 1.6\%. For Experiment 1b, the rates at which inconsistencies were correctly resolved was $53.5 \%$

\footnotetext{
${ }^{2}$ We also examined the MCS algorithm without normalizing the confidence ratings and the results are consistent with those found using normalization. These results are presented in the Supplement.
} 
and $54.0 \%$ for blast and non-blast cells respectively, leading to a reduction of false alarms by $0.9 \%$ and misses by $0.8 \%$. To test if this difference was significant, we conducted a 2 (algorithm: average performance, max confidence slating) x 2 (classification: blast, non-blast) repeated measures ANOVA with accuracy as the dependent variable. Specifically, we were interested in the effect of algorithm and the interaction of algorithm and classification. For Experiment 1a, there was a main effect of algorithm, $F(1,33)=15.37, p=0.0004$. The interaction between algorithm and classification was not significant, $F(1,33)=0.22, p=0.6455$. For Experiment $1 \mathrm{~b}$, once again, there was a significant main effect of the algorithm, $F(1,30)=8.12, p=0.0078$. There was no significant interaction with classification, $F(1,30)=0.003, p=0.9505$. This suggests that the improvements in Experiments 1a and 1b occurred across both blast and non-blast cells.

For Experiment 2, the accuracy increased by $2.2 \%$ to $73.7 \%(t(21)=3.5, p=0.0020)$ by resolving $59.6 \%$ of the inconsistencies correctly. We observed an improvement even without the normalization step. However, this improvement was not significant (MCS - 72.5\%, AR- 71.6\%, $\mathrm{t}(21)=1.16, p=0.2568)$.

For Experiment 2, to examine the MCS algorithm in more detail, we conducted a 2 (algorithm: average performance, max confidence slating) x 2 (classification: blast, non-blast) repeated measure ANOVA with accuracy as the dependent measure. We observed a significant main effect of algorithm $(F(1,21)=12.4, p=0.0020)$. The interaction with classification was not significant $(F(1,21)=0.2, p=0.6789)$. This suggests that the algorithm is equally effective for blast and non-blast images for experts. These results shows that the maximum confidence slating algorithm is effective for both novices and experts for both cell types.

Next, we examined the maximum confidence slating algorithm for two different individuals. We randomly paired two individuals together and applied the algorithm to their first responses. As a baseline, we compared it to the average first response performance of the dyad. This was calculated as the average of the mean performance of the dyad. The MCS-between accuracy was significantly greater than the average performance for both novice experiments. It increased by $3.9 \%$ to $70.0 \%$ in Experiment $1 \mathrm{a}(t(16)=5.8, p<0.0001)$ and by $3.5 \%$ to $70.0 \%$ in 
Experiment $1 \mathrm{~b}(t(14)=5.2, p=0.0001)$. We observed that for Experiment $1 \mathrm{a}$ and $1 \mathrm{~b}, \mathrm{MCS}$ improved performance even without the normalization step (Exp. 1a: MCS - 69.1\%, AR - 66.7\%, $t(16)=2.20, p=0.0429$; Exp. 1b: MCS - 70.0\%, AR- 66.5\%, $t(14)=3.57, p=0.0030)$. For experts in Experiment 2, there was a larger improvement in accuracy using normalization, increasing by $6.9 \%$ to $78.5 \%(t(10)=3.1, p=0.0104)$. For Experiment 2 , without the normalization step, we see an improvement. However, this improvement is only marginally significant $(\mathrm{MCS}-75.9 \%$, AR- 72.4\%, $t(10)=1.93, p=0.0827)$. These results are summarized in Table2,

Table 2

The mean performance of each algorithm for Experiments $1 a, 1 b$, and 2.

\begin{tabular}{llll}
\hline Algorithm & Exp. 1a & Exp. 1b & Exp. 2 \\
\hline Average Response - Within & $66.1 \%$ & $66.5 \%$ & $71.6 \%$ \\
Max. Conf. Slating - Within - Norm. & $67.4 \% *$ & $67.4 \% *$ & $73.7 \% *$ \\
Max. Conf. Slating - Within - No Norm. & $67.6 \% *$ & $67.3 \% *$ & $72.5 \%$ \\
\hline Average Response - Between & $66.7 \%$ & $66.5 \%$ & $72.4 \%$ \\
Max. Conf. Slating - Between - Norm. & $70.0 \% *$ & $70.0 \% *$ & $78.5 \% *$ \\
Max. Conf. Slating - Between - No Norm. & $69.1 \% *$ & $70.0 \% *$ & $75.9 \%$ \\
\hline Note. *Significant improvement as compared to average response with p<0.01
\end{tabular}

\section{Discussion}

We observed that the MCS-within algorithm improved performance for both the novice experiments. Our experiments were designed to allow for a within-subject comparison before and after the application of the algorithm. Hence, we are able to achieve high significance (small p-values) in this within-subject comparison since the algorithm resulted in an improvement for most subjects. First, this is important and shows that participants not only learned cancer cell classification, but also developed their metacognitive skills in the brief training phase. Second, MCS reduced both false alarms and misses. Third, the WoIC effect occurred in the absence of additional manipulations to increase the diversity of responses. We observe that the improvement in performance without the normalization step was not significant for the experts. This might be partially explained by the smaller sample size and shorter task. However, this also might be due to 
differences in the confidence judgments provided by the experts in the two frames. We discuss this point further in the General Discussion. Finally, it is important to note that the improvement in MCS algorithms was greater when we used different individuals than when we used the same individual.

We also observed that the MCS-within algorithm improved performance for the experts. This shows that despite their expertise, even experts might respond differently for the same image and that the metacognitive information that they provide can be used to resolve these inconsistencies. Further, we observed that the algorithm reduced both false alarms and misses to improve performance. Finally, we observed that using two different experts resulted in an even larger improvement in performance. This is larger in magnitude than the improvement for the novice experiments and can be explained by the greater inter-rater disagreement observed in the expert data, as discussed in Experiment 2.

\section{Wisdom of the Crowds Algorithms}

In this section, we present the WoC algorithms. First, we describe general WoC algorithms that aggregate responses only based on the classification decisions made by the group of decision makers and not their confidence judgments. Second, we describe WoC algorithms that are based on metacognitive judgments. For our analyses, we will vary the number of participants $(n)$ used to form a group. For some algorithms, having an even number of participants would require us to randomly break ties. Since we were interested in the trends and comparisons among algorithms, we only used an odd number of participants to form the groups. For a given number of people, we randomly select a subset of $n$ participants from our data and apply the algorithms. Finally, we calculate the accuracy. We repeated this process 1000 times and report the average accuracy.

\section{General WoC Algorithms}

Simple Voting - One Response ( $\mathrm{SV}-\mathrm{O})$ : For each image, for each person, the first classification response is used to calculate the final response. All these responses are pooled 
together, and the modal response (most common) response is taken as the final response. Any ties are broken randomly.

Simple Voting - Both Responses (SV-B): For each image, for each person, both classification responses are used to calculate the final response. As in SV-O, all of these responses are pooled together, and the modal response is taken to be the final response. Any ties are broken randomly.

\section{Metacognitive (Confidence) Judgment based Algorithms}

In this section, we describe WoC algorithms where each decision is weighted by the confidence on that image.

Confidence Weighted Voting - One Response ( $\mathrm{CWV}-\mathrm{O})$ : The scores were defined as the sum of all of the individual-level scaled confidence judgments for responses of a particular type (i.e., blast or non-blast). The response with the maximum score was taken to be the final response.

Confidence Weighted Voting - Both Response (CWV-B): This is similar to CWV-O except that we use both responses. We calculated the score of both responses using the method mentioned above, using both the decisions and their corresponding confidence judgments. The response option with the maximum score was taken to be the final response.

Confidence Slated Voting (CSV): For each participant in the group, we first slated each classification response to the more confident response, as described above. This becomes the individual's final response. The individual's response with lower confidence judgment is discarded. These responses were then used to make a final group response using the process of confidence weighted voting as described in CWV-O).

\section{Variants of Confidence Weighting}

We use confidence judgments with and without a normalization step. For the non-normalized confidence judgments, we use the confidence judgments provided by the $n$ participants in the group. We also test the log-odds transformation of the confidence judgments to 
weight decisions. For normalizing the judgments, we use the following procedure so that every individual's decision has the same weight. We first calculate the mean and standard deviation of the group's confidence judgments before aggregation. We then re-scale the confidence judgment of every individual with the group's mean and standard deviation so that they have the same mean and standard deviation.

Log Odds Weighting: We consider analogous algorithms to CWV-O, CWV-B and CSV, where instead of summing the confidence judgments, we sum the log odds of the confidence judgments.

The log-odds transformation can be visualized in the Supplement. With the log-odds transformation, a confidence judgment of $50 \%$ receives a weight of 0 , and hence is viewed as uninformative and is discarded. Highly confident decisions are weighted much more heavily than less confident decisions with the log-odds transformation as compared to using these judgments

directly without the transformation. For example, if a decision maker gave a confidence judgment of $90 \%$, then it would receive a weight of 2.20 . However, if a decision maker gave a judgment of $60 \%$, then the assigned weight would be 0.41 . This would imply that one would need about 6 decisions made with $60 \%$ confidence to overrule one decision made with $90 \%$ confidence.

If a decision maker made a decision with $100 \%$ confidence, then the log-odds transformation would give it an infinite weight. To avoid such cases, we consider an extra parameter, tolerance $(t)$ to cap confidence. A tolerance of $t$ would cap confidence at the (1- $t$ ) level. In this paper, we consider a tolerance of $t=0.05$ and cap confidence at the $95 \%$ level. We tested out different tolerance values and report our results in the Supplement. In the Supplement, we observe that using a tolerance that is very low hurts performance.

\section{Results}

As described above, we randomly chose a subset of participants from the pool of participants for each experiment. We then applied the WoC algorithms to this group. We report the average performance of the algorithms after repeating this process 1000 times. We display our 
results for general WoC and metacognition based WoC algorithms for different group sizes in Figure 4, As shown in the figure, across all algorithms and experiments, the performance improved with group size. The rise is more dramatic in the beginning, with smaller group sizes, after which it starts tapering off.

For Experiment 1a, we report our results for group size $n=7$ in Table 3 . We make the following observations. The accuracy for simple voting with one response was $77.6 \%$. This increased to $80.7 \%$ when we used two responses. When we weighted by confidence (without the normalization step) and only used the first response, the accuracy was $78.3 \%$. Weighting both responses with confidence gave us an accuracy of $81.8 \%$. This shows that using two responses is better than using one. Also, weighting by confidence was better than not weighting by it. We also observe that the results were similar when decisions were combined after the normalization step and with the log-odds transformation of the confidence judgments.

We saw a similar pattern for Experiment 1b. However, the difference between the algorithms with one and two responses was smaller. Using only the first response made by the participant, resulted in an accuracy of $78.7 \%$, and using both responses resulted in an accuracy of $80.0 \%$. Without normalization, weighting the first response with confidence gave us an accuracy of $79.2 \%$, while using both responses increased the accuracy to $81.0 \%$. Once again, confidence slating came in the middle with an accuracy of $79.3 \%$. These results were similar when we weight by normalized confidence and with the log-odds transformation.

For Experiment 2, Table 3 shows the general WoC and meta-cognition based WoC algorithms for groups with 7 participants. Using only one response gave us an accuracy of $86.2 \%$ and using two responses gave us an accuracy of $86.9 \%$. Weighting the one response with confidence gave us a slight improvement to $86.7 \%$ and weighting both responses with confidence increased it to $87.9 \%$. Weighting decisions with the log-odds transformation of confidence performed similarly. Normalization seemed to hurt performance for the confidence based algorithms especially when the log-odds transformation was used. Confidence slating did poorly and came in the middle at $85.5 \%$. 
We wanted to compare the performance of different algorithms on novices and experts. To this end, we restricted the decisions of novices from Experiment 1a to the images that were used in Experiment 2. We compared the performance of novices and experts - Confidence Weighted Voting without normalization (see Figure 4 panel D). For one decision maker, the average performance was $62.2 \%$ and $73.4 \%$ for novices and experts respectively. We also wanted to know how many novices can match the performance of an expert. Increasing the group size to 5 boosts the accuracy by $11.1 \%$ to $73.3 \%$ for novices, matching the performance of the experts. However, using 5 experts improved their accuracy by $12.2 \%$ to $85.7 \%$ for the experts. Hence, as in Figure 4 . approximately 5 novices matches the performance of one expert.

\section{Table 3}

Results of the different Wisdom of the Crowd (WoC) algorithms when groups of 7 decision makers are considered.

\begin{tabular}{|c|c|c|c|}
\hline Algorithm & Exp. 1a & Exp. 1b & Exp. 2 \\
\hline First Response (1 Person) $^{a}$ & $66.5 \%$ & $67.0 \%$ & $73.0 \%$ \\
\hline Simple Voting - First Resp. & $77.6 \%$ & $78.7 \%$ & $86.2 \%$ \\
\hline Simple Voting - Second Resp. & $77.5 \%$ & $77.5 \%$ & $84.0 \%$ \\
\hline Simple Voting - Both Resp. & $80.7 \%$ & $80.0 \%$ & $86.9 \%$ \\
\hline Conf. Weight Voting - First Resp. - No Norm. & $78.3 \%$ & $79.2 \%$ & $86.7 \%$ \\
\hline Conf. Weight Voting - First Resp. - Norm. & $78.2 \%$ & $79.1 \%$ & $86.3 \%$ \\
\hline Conf. Weight Voting - First Resp. - Log Odds - No Norm. & $79.4 \%$ & $80.4 \%$ & $88.6 \%$ \\
\hline Conf. Weight Voting - First Resp. - Log Odds - Norm. & $79.4 \%$ & $79.5 \%$ & $86.7 \%$ \\
\hline Conf. Weight Voting - Second Resp. - No Norm. & $78.2 \%$ & $78.0 \%$ & $84.0 \%$ \\
\hline Conf. Weight Voting - Second Resp. - Norm. & $78.2 \%$ & $77.8 \%$ & $83.7 \%$ \\
\hline Conf. Weight Voting - Second Resp. - Log Odds - No Norm. & $77.7 \%$ & $78.4 \%$ & $83.8 \%$ \\
\hline Conf. Weight Voting - Second Resp. - Log Odds - Norm. & $78.3 \%$ & $77.6 \%$ & $83.2 \%$ \\
\hline Conf. Weight Voting - Both Resp. - No Norm. & $81.8 \%$ & $81.0 \%$ & $87.9 \%$ \\
\hline Conf. Weight Voting - Both Resp. - Norm. & $82.1 \%$ & $\mathbf{8 0 . 8 \%}$ & $87.0 \%$ \\
\hline Conf. Weight Voting - Both Resp. - Log Odds - No Norm. & $81.3 \%$ & $81.0 \%$ & $88.3 \%$ \\
\hline Conf. Weight Voting - Both Resp. - Log Odds - Norm. & $81.9 \%$ & $80.5 \%$ & $86.7 \%$ \\
\hline Conf. Slated Voting - No Norm. & $80.2 \%$ & $79.3 \%$ & $85.5 \%$ \\
\hline Conf. Slated Voting - Norm. & $79.5 \%$ & $79.4 \%$ & $86.7 \%$ \\
\hline Conf. Slated Voting - Log Odds - No Norm. & $79.9 \%$ & $79.8 \%$ & $85.7 \%$ \\
\hline Conf. Slated Voting - Log Odds - Norm. & $79.7 \%$ & $79.1 \%$ & $86.6 \%$ \\
\hline
\end{tabular}

Note. The top 4 best performing algorithms for each experiment are in bold. All but one of them are confidence-weighted weighting with two responses.

${ }^{a}$ The average accuracy using only the first response for a group size of one is provided as a baseline comparison. 


\section{Discussion}

When aggregating responses across a group of people, we observed that two responses per person were better than one response per person. Further, weighting responses by confidence is better than simple voting for all experiments. We also observed that the second response did not substantially exceed the accuracy of the first response for all three experiments. This is important since the improvement in performance due to aggregation cannot be explained by higher performance on the second block. In fact, for Experiment $1 \mathrm{~b}$ and Experiment 2, the accuracy might be slightly lower on the second block. This might be due to fatigue or an inability to follow the change in instruction across the two blocks despite the practice block.

We also observed that confidence weighting on a single response does not outperform taking a second response from the same individual and using simple voting (i.e., CWV-O performs worse than SV-B). This shows that having two responses per individual instead of one improves performance. Overall, the best performing algorithm is where both the responses are weighted by the confidence. It is worthwhile to point out that even though CWV-B outperforms $\mathrm{SV}-\mathrm{B}$, the improvement is only marginal and comes at the cost of the additional time it takes to collect the confidence responses and then the extra effort of normalizing them. The confidence slating algorithm does not outperform the weighting algorithm. Confidence slating overrides the response with lower confidence. On the other hand, confidence weighting considers both responses and their confidences. The pattern of results indicates that overriding the low confident decision may lead to a suboptimal use of information. These results are consistent with the Brier decomposition results presented earlier at the individual level, where we showed that averaging confidence as opposed to taking the more confident response resulted in judgments with higher resolution that are more reliable with better Brier scores.

When we weight by log-odds we observe that the results are largely similar to when we weighted decisions by the confidence judgments directly. In all three experiments, it increased accuracy when the first decision was weighted by confidence. However, the results do not replicate when the second decision or both decisions are weighted by the log-odds of the 
confidence. We also observe similar results when we look at the confidence slating algorithm. Hence, we interpret this as evidence that weighting the log-odds of the confidence judgments performs similarly as using the confidence judgments directly. We discuss this point further in the General Discussion. Finally, we also observe that algorithms using normalized and non-normalized confidence judgments perform similarly.

\section{General Discussion}

In this paper, we explored different methods for aggregating repeated decisions to improve accuracy in the context of medical image decision making. We showed that it is possible to use findings from the WoC literature to improve diagnostic accuracy, with the accuracy of several novices approaching that of a single expert. We also showed a WoIC effect where it is possible to improve an individual's accuracy by combining repeated decisions about the same image using maximum confidence slating. However, aggregating decisions from different decision makers can appreciably improve the decision accuracy.

The MCS algorithm works by exploiting people's metacognitive processes, namely their ability to judge the accuracy of their responses (Fleming et al., 2012; Griffin \& Tversky, 1992; Yeung \& Summerfield, 2012). We found that the MCS algorithm improved performance in all of our experiments for both cell types, suggesting that confidence judgments can meaningfully solve the conflict of inconsistent decisions. For experts, these results are consistent with the findings of Litvinova et al. (2022) where the authors recommend taking the more confident response to solve an inconsistent pair of responses. However, even with the experts, there are several important differences in our approach. First, Litvinova et al. (2022) used a different set of experts and a different set of images. They used data from radiologists examining mammograms and physicians examining spinal scans (i.e., visual search tasks) which are different from pathologists examining white blood cell images (i.e., complex categorization task). Second, the data that was used by Litvinova et al. (2022) used expert decisions that were separated by a few months. This reduces it's practical applicability because diagnostic decisions are time sensitive. In our experiment, the 
two decisions were separated by a few minutes. While this might raise a concern about participants remembering their decisions, Trueblood et al. (2018) showed that participants' performance on a set of images used in the training trials was indistinguishable from the performance on novel stimuli, suggesting that participants cannot remember the original label on the image.

For the first time, we show that despite receiving very limited training, novices can meaningfully report on their confidence in medical image decision tasks, which is important for the practical use of crowd sourcing medical decisions. Interestingly, we notice that experts and novices have similar reliability scores and Brier scores in the Murphy decomposition of their raw and normalized confidence judgments, suggesting that novices effectively used the confidence scale. The only difference was in their ability to resolve cancer and non-cancer cells. We note this might be partially explained by the differences in task structure which can impact confidence judgments (Rahnev \& Fleming, 2019). Further, we also note that we asked participants to provide a confidence judgment on a scale of $50 \%-100 \%$ where $50 \%$ meant guessing and $100 \%$ completely certain. While this probably elicited subjective probability judgments, we did not explicitly ask them to provide subjective probability judgments. Our results also indicate that using a WoC of minimally trained novices, might be able to achieve expert-like performance on medical image classifications. We note that higher rates of inconsistencies and corresponding higher rates of improvement are more prominent in Experiment 1a than Experiment 1b. This suggests that eliciting more varied responses might be useful in achieving a larger wisdom of the inner crowd effect. Hence, we see that the dialectic bootstrapping technique used in our paper meaningfully elicited more diverse responses. However, as mentioned in the discussion above, it is not clear which of the two manipulations were required to bring about this change. We also showed that the MCS algorithm works better when we use the decisions from two different decision makers. This finding holds true for novices and experts and can be explained by the observation that the intra-rater disagreement is less than the inter-rater disagreement.

Research on confidence has mainly focused on two aspects - resolution and calibration 
(Koriat, 2012a). Resolution refers to internal consistency such that the confidence judgments of an individual can be used to discriminate between accurate and inaccurate responses (Liberman \& Tversky, 1993). Calibration refers to providing confidence judgments that correspond to the probability of providing an accurate response. For the MCS-Within algorithm, confidence judgments need not be calibrated but they need to be able to resolve correct and incorrect responses. We observe that for novice participants, the MCS algorithm is effective even without the normalization step. However, this did not generalize to the expert participants, where the improvement due to MCS was not significant when omitting the normalization step. This might be due to us having lower power for the expert task because we had fewer participants and a shorter task. However, as we see in the Supplement, there might be a difference in the way experts use the confidence scale in the two frames ("Is this cell cancerous?" and "Is this cell not cancerous?"). These differences were not significant for novices. Hence, it is possible that the two frames elicited slightly different confidence scales for experts. Normalization fixes some of these biases and might play an important role in the effectiveness of MCS.

For the MCS - Within algorithm, it is sufficient if the confidence judgments are able to resolve correct and incorrect responses. It is not necessary for these judgments to be well-calibrated. However, when combining the decisions of different participants with potentially different confidence calibrations, an overconfident decision maker might dominate all decisions. In our experiments, we normalized the confidence using the Self Consistency Model (Koriat, 2012a) of each participant before combining their decisions. In this paper, we observe that without this normalization step, the MCS algorithm improves performance for novices and experts. However, the improvement for the experts is not significant. This might be partially due to having a smaller group size and fewer trials in the expert group. However, this might also indicate that it is important to normalize the decisions before aggregation to account for individual differences in the use of the confidence scale. This finding has important consequences for using a confidence-based aggregation algorithm since normalizing requires several decisions made by the same decision maker which, might not be possible in clinical practice. Previously, 
MCS has been applied to a visual perception, general knowledge (Koriat, 2012a), and a visual search task (Bang et al., 2014; Koriat, 2012b). In Bang et al. (2014), the authors show that the difference in improvement in performance due to MCS in the absence of normalization is significant but small. In their set-up participants had access to each other's confidence judgments at the end of each trial during the experiment. This might have allowed them to arrive at a jointly-consistent scale. It is possible that in a clinical setting, a dyad of medical professionals working in the same facility might be able to achieve a jointly consistent confidence scale by observing their partners' responses on other cases. As mentioned in the introduction, WoC algorithms are commercially deployed in the real world to label large datasets to train artificial intelligence algorithms (Alialy et al., 2018; Dissanayake et al., 2019; Ørting et al., 2020; Press, 2021). In these systems, medical decisions are collected from a distributed network of individuals who minimally interact with each other (Press, 2021). In such a system, since each individual contributes several decisions, one can normalize confidence scales before aggregation.

Our results show that the gains made by improving the performance of a single person using WoIC techniques, can be channeled into improving the performance of the crowd. In this regard, we note that pooling both the decisions made by an individual is better than using only one of them. We also note that further improvements to accuracy are obtained by weighting responses by their confidence. Further, weighing with confidence gives us better results than considering only the more confident response. These results also parallel the Brier decomposition in the earlier section where, even at the individual level, using confidence leads to decisions with better resolution as well as being more reliable and with a lower Brier score. Further, averaging confidence leads to better resolution and more reliable judgments than maximizing confidence. This result is consistent with the recommendation made by Litvinova et al. (2020), where the authors recommend that one should average responses instead of considering only the more confident one. This suggests that confidence slating loses meaningful information contained in the confidence judgments of the two responses. These patterns are once again similar for experts and novices. 
The improvement in performance observed in our experiments is by no means trivial since the relationship between group size and accuracy of the crowd varies across different types of binary decision tasks (Lee \& Lee, 2017). In practical applications, it is important to weigh the increase in accuracy with the additional resources required to collect confidence ratings or to gather multiple votes on each image or both. In our data, the median response time for confidence judgments was about the same as the median response time for the second response. Even though the time needed to collect confidence judgments and second responses was similar in our tasks, confidence judgments might be more difficult to implement in real world situations. Thus, it might be better for one to collect two decisions rather than one with a confidence judgment since we find that the addition of the confidence weighting only improves accuracy marginally.

In our paper, we used a simple model so that confidence judgments had the same mean and standard deviation for all participants. We then combined responses by directly weighing them by their confidence judgments. We observe that the log-odds aggregation performs similarly to summing the confidence judgments. This suggests that different ways of weighting decisions by confidence might lead to similar results, especially with larger group sizes. However, we note that it is unclear what is the optimal way to aggregate decisions. Future work might examine re-calibrating confidence judgments to optimally aggregate decisions (Attali et al., 2020; J. Baron et al., 2014; Steyvers et al., 2014; Turner et al., 2014). In this work, we used the crowd to create a "final" decision. In future work, one might design and test WoC algorithms that generate a confidence judgment associated with the crowd's decision based on the individual confidence judgments that comprise it. There are different ways of estimating the group's confidence judgment based on different theoretical principles. For example, to aggregate two identical decisions (say cancerous) made with $70 \%$ confidence, the final decision could be estimated to have $70 \%$ confidence since both the decision makers agree on the uncertainty of their estimate. Alternatively, one could increase confidence in the final answer due to the consensus on the decision being cancerous. In algorithms that estimate the confidence judgment along with the decision, issues of re-calibration and weighting might have a larger impact. 
With a rather cheap and simple self-report of a numerical value of confidence, one can improve the accuracy of decision making across experiments. The original finding of Koriat (2012b) was in response to Bahrami et al. (2010) to show that a simple confidence judgment can be used to communicate between individuals for effective joint decision making in the place of social interaction. We note that the effectiveness of WoC is in direct opposition to the 'group-think' phenomenon where decisions made in a cohesive group can have disastrous outcomes (R. S. Baron, 2005; Esser \& Lindoerfer, 1989; Janis, 1973). Combining decisions thereby might depend on the underlying task conditions and the protocol that is used to combine them. In a comparison between dyadic interaction and maximum confidence slating, Koriat (2015) and Tullis and Goldstone (2020) showed that dyadic interaction might be more effective than maximum confidence slating for perceptual decision making problems and educational reasoning problems (eg. physics problems) respectively. Therefore, for some medical image decision tasks, it might be beneficial to allow for social interaction of decision makers. Future research might focus on designing specific protocols so that two (or more) decision makers can communicate information to one another in the case of medical decisions. Such protocols will likely need to be tailored for specific medical decision problems since the kind of observations required for one problem might be very different from another.

\section{Constraints on Generality}

We observe that in our task, we asked participants to provide confidence judgments on a half-range scale - where participants provided confidence judgments on a scale of 50-100. We note that there are different ways of eliciting confidence judgments such as using a 7 point Likert scale, full-range confidence scales - where participants provide their confidence judgment on a scale of 0-100 etc. We note that different response modes can sometimes result in differences in calibration and result in different overconfidence and underconfidence biases (Olsson, 2014; Rahnev \& Fleming, 2019). Hence, WoC algorithms might depend on the scale used to elicit confidence responses. However, we do not expect the effectiveness of the algorithms presented in 
the paper to depend on the response mode since they do not hinge on well calibrated responses.

One of the other limitations of this study is that it uses only one type of images Wright-stained white blood cells. For other images, the cognitive process involved might be very different. For example, with a lung nodule detection task, the problem is one of visual search (Drew et al., 2013). Thus, we believe our results are reproducible with images similar to Wright-stained white blood cells, but may not generalize to other types of medical images. Second, our data from experts is limited. Despite this limitation, we could make some interesting inferences, including showing that WoIC and WoC methods work for our expert population. Third, due to time constraints with expert participants, the experimental design between experts and novices was slightly different, leading to an imperfect point of comparison. For example, the reduced time between the two decisions in Experiment 2 might have lead to a lower intra-rater disagreement and decreased the diversity of responses for experts. Thus, our results could be viewed as a lower bound on the effectiveness of the WoIC algorithms for experts.

Another limitation of this study is that we only examined the case where blast cells occur at $50 \%$ prevalence. We note that our task is a simplified version of the actual task performed by medical professionals. In the real-world task, a morphological classification of blood cells (e.g., as blasts and various types of non-blast cells), is the first step in diagnosing malignant blood diseases. In many clinical settings (such as a large hospital), this step is first performed by an automated image analyzer in combination with a knowledgeable human observer (often a medical technologist). The automated system will pre-classify images and present grouped images to the human observer. Thus, the prevalence rate will be quite high for grouped images (e.g., if the machine creates a "blast" cell group, most of the images in that group will be blast cells). While automated systems are routinely used in many clinical settings (particularly in the United States), not all laboratories have access to such technology and rely on manual review with a microscope. In this case, prevalence will not be manipulated by the machine. In our task, we use $50 \%$ prevalence since in the real-world, prevalence can depend on the way images are reviewed. Future research could examine the algorithms and approaches discussed here when the prevalence rate of 
blast cell images is manipulated. We also refer the reader to Trueblood et al. (2021) for a more detailed discussion of the blast identification task when prevalence is manipulated.

\section{Conclusion}

In this paper we explored how to leverage metacognitive processes in the form of confidence judgments to improve the accuracy of medical image decision making with novices and experts. At the individual level, the maximum confidence slating algorithm, which considers the more confident response as the final one, improves the accuracy of both novices and experts. This shows that metacognitive information can be collected and utilized when evaluating medical image decisions regardless of decision makers' training. Further, the gains made by using different decision makers surpass the gains made by using repeated decisions from the same person. Further, we can form larger units of decision makers to dramatically improve the accuracy of novices and experts. This improvement can be boosted by using findings from the WoIC level, where using two responses and weighting them by confidence can further improve performance. In sum, our results show that self-reported confidence can be robustly used to combine repeated decisions made by the same individual, by two different individuals, and by larger groups of participants for both novices and experts. 


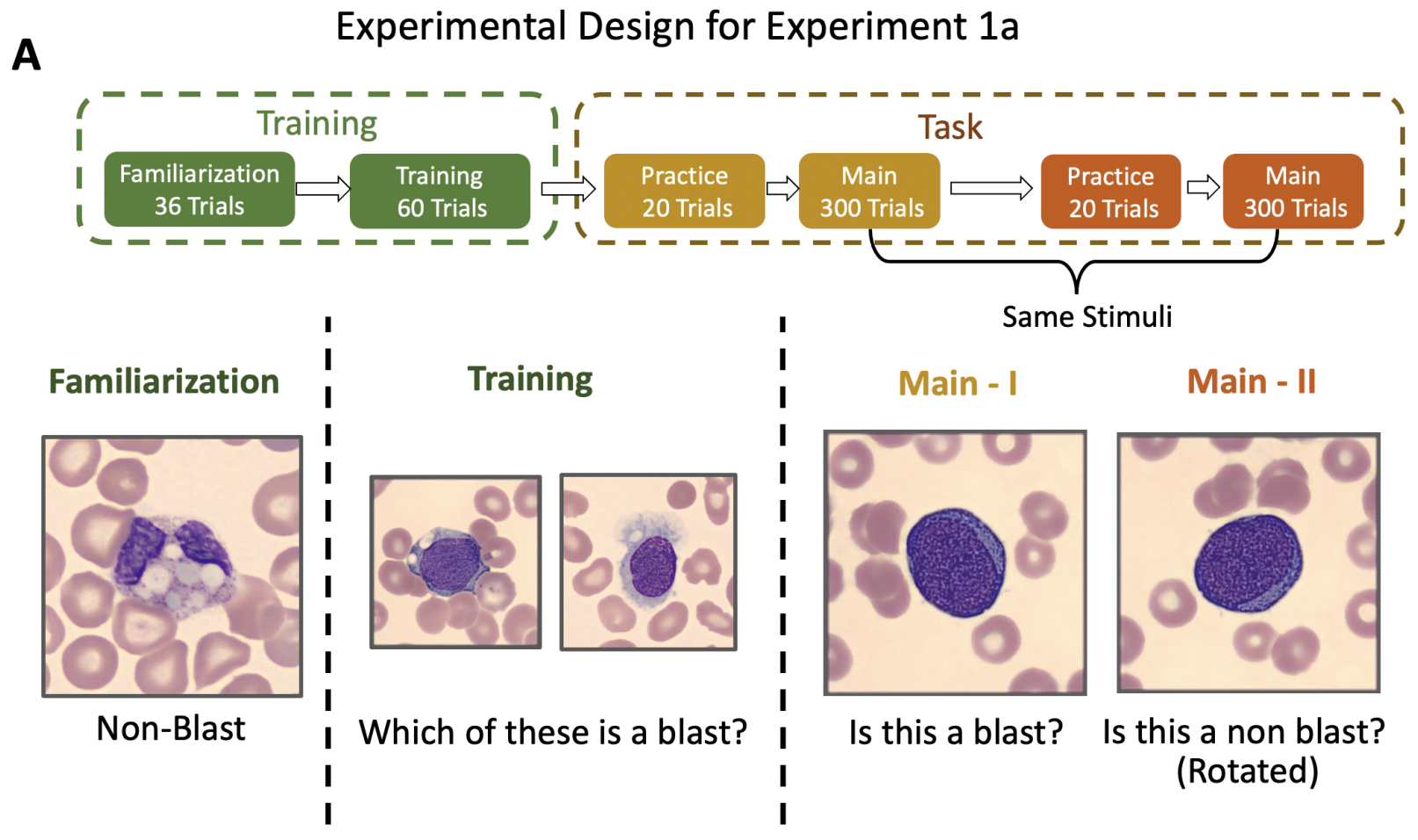

B

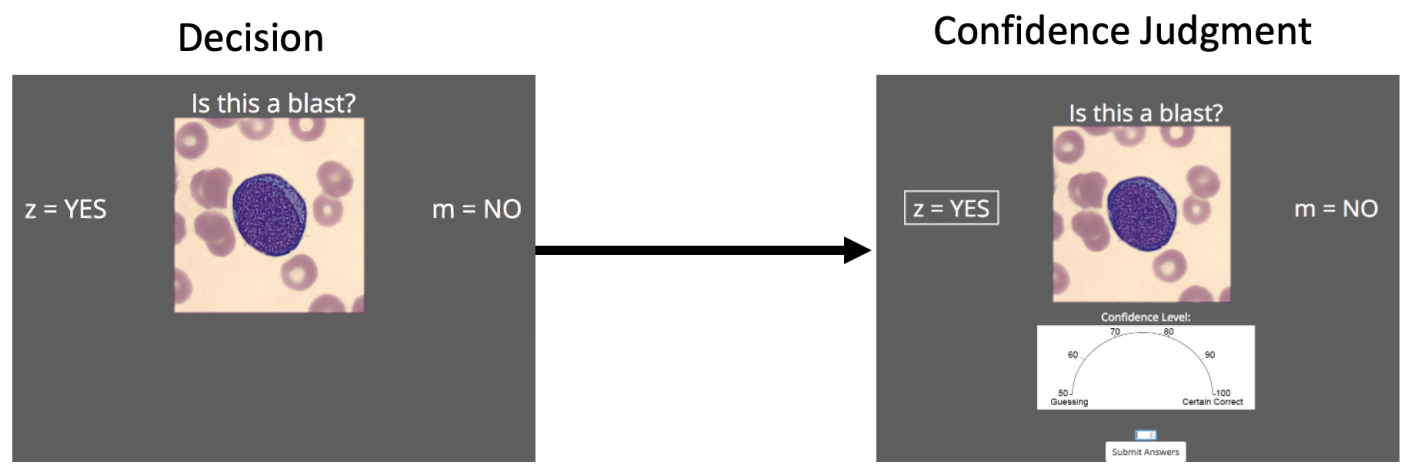

\section{Figure 1}

Panel A illustrates the structure of Experiment 1a. Participants first completed a brief training phase consisting of two blocks where they learned to discriminate between blast and non-blast cells (shown in green). In the main task, two responses were collected for each participant on each of the 300 images. In the first pass (shown in yellow), participants were presented with the image of a cell and were asked the question 'Is this a blast?'. In the second pass (shown in orange), the same image was rotated and participants were asked the question 'Is this a non-blast?'. Each set of main trials was preceded by practice trials to familiarize participants with the interface. Experiment $1 b$ was similar to Experiment 1 a except that the same question was asked in both of the main blocks (i.e., 'Is this a blast?') and images were not rotated. Experiment 2 was a shorter version of Experiment la and was designed for expert participants. Panel B shows the two parts of every trial in the main task. In the first part, participants decided whether a cell was a blast or not. In the second part, participants indicated their confidence on a scale of 50-100, where 50 was 'guessing' and 100 was 'certain correct'. 
A

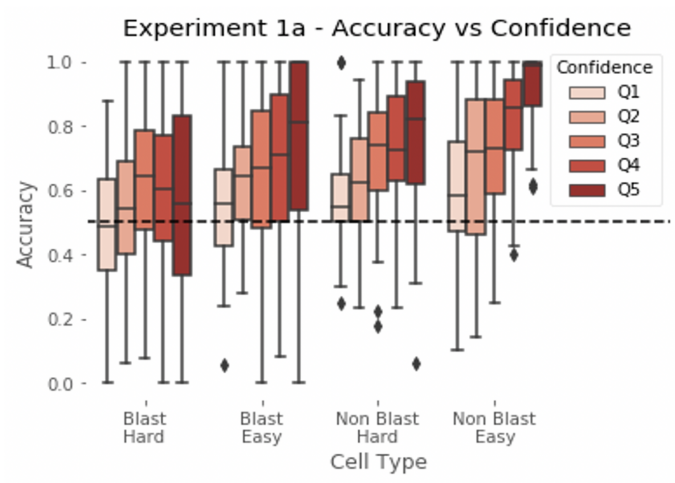

C

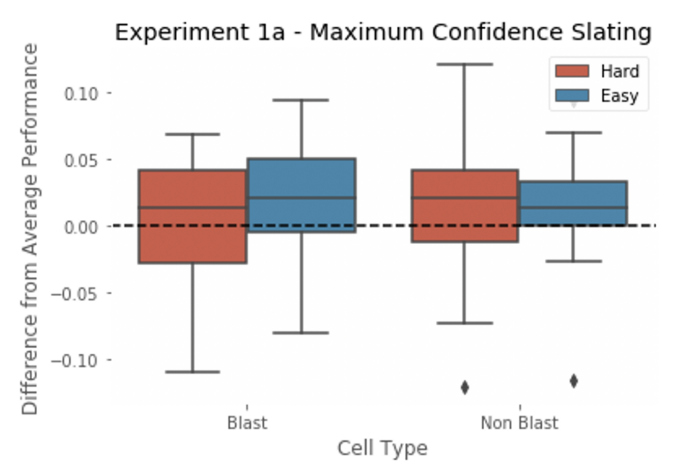

B

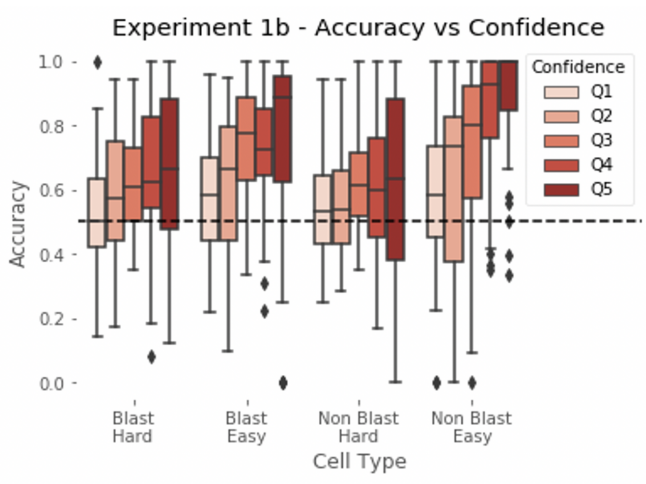

D

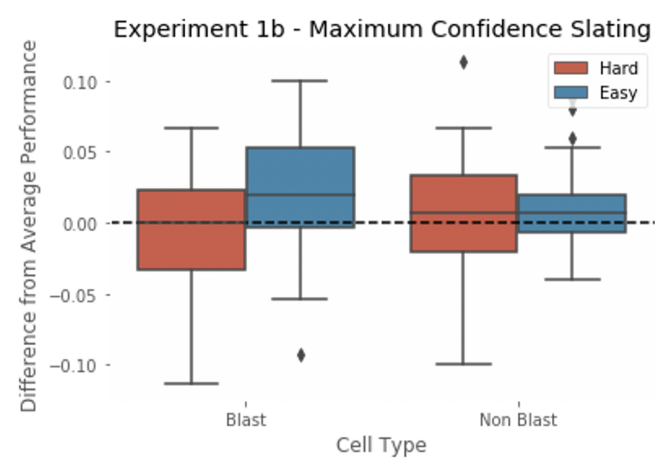

\section{Figure 2}

Panel A (B) shows the relationship of confidence with accuracy, cell type, and difficulty for Experiment $1 \mathrm{la}(\mathrm{lb})$. We observe a positive relationship between confidence and accuracy. Panel $C(D)$ plots the difference between average accuracy and the accuracy obtained using the maximum confidence slating algorithm. We observe that for most cell types accuracy increases when using maximum confidence slating. 
A

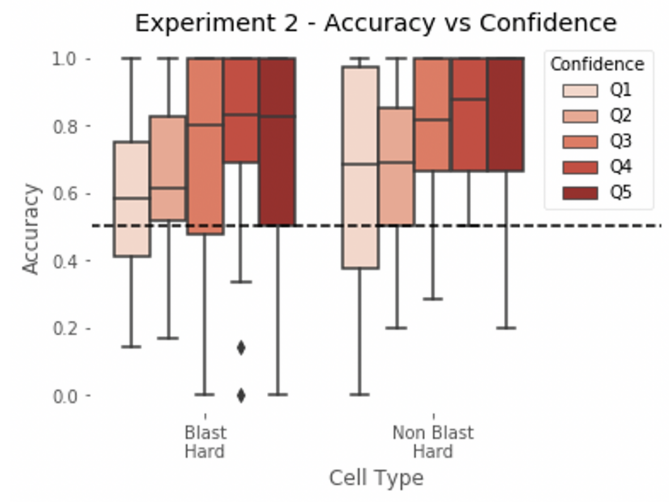

B

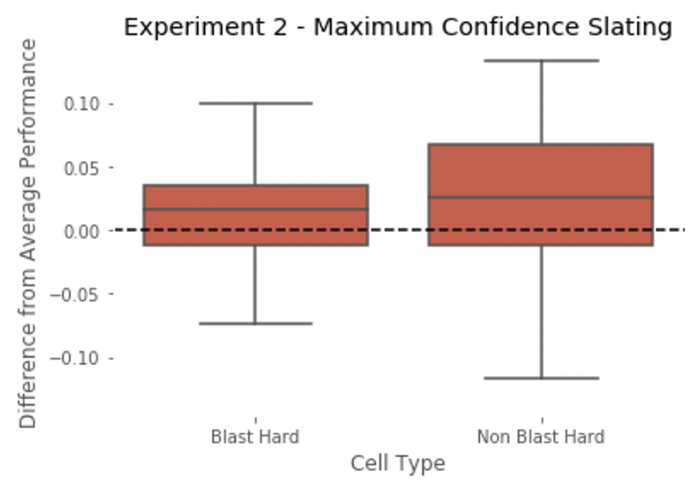

\section{Figure 3}

Panel A shows the relationship of confidence with accuracy and cell type for Experiment 2. We observe a positive relationship between confidence and accuracy. Panel B plots the difference between average accuracy and the accuracy obtained from the maximum confidence slating algorithm. We observe that for both cell types accuracy increases when using maximum confidence slating. 
A

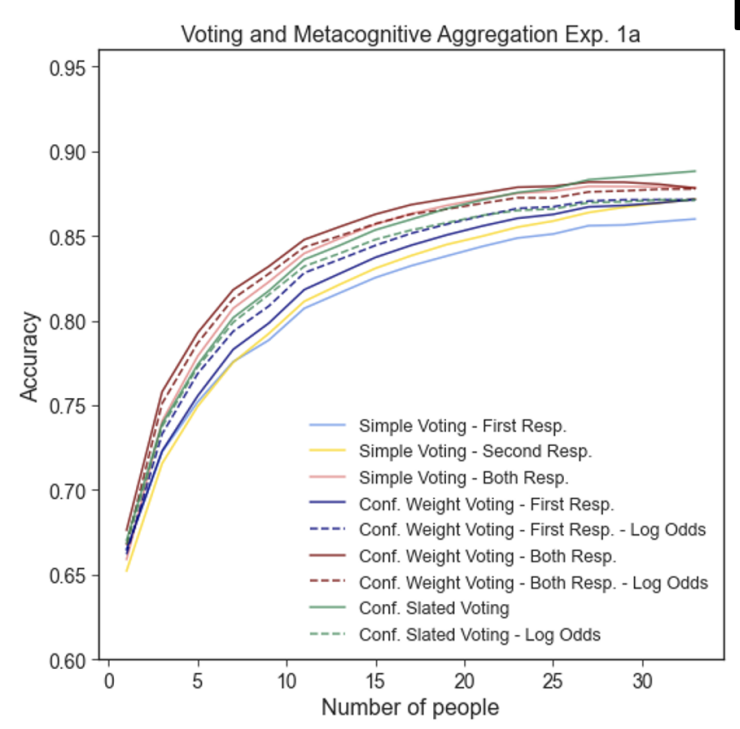

C

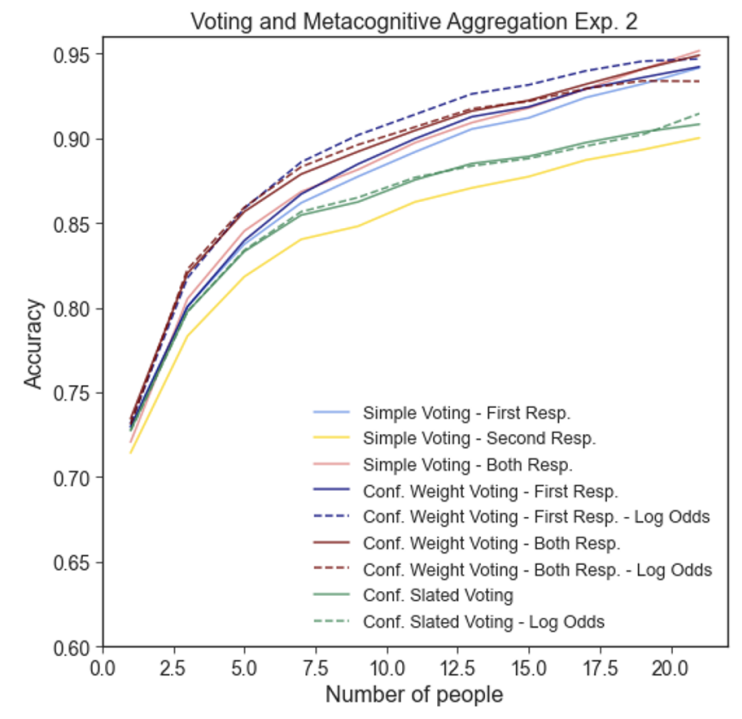

B

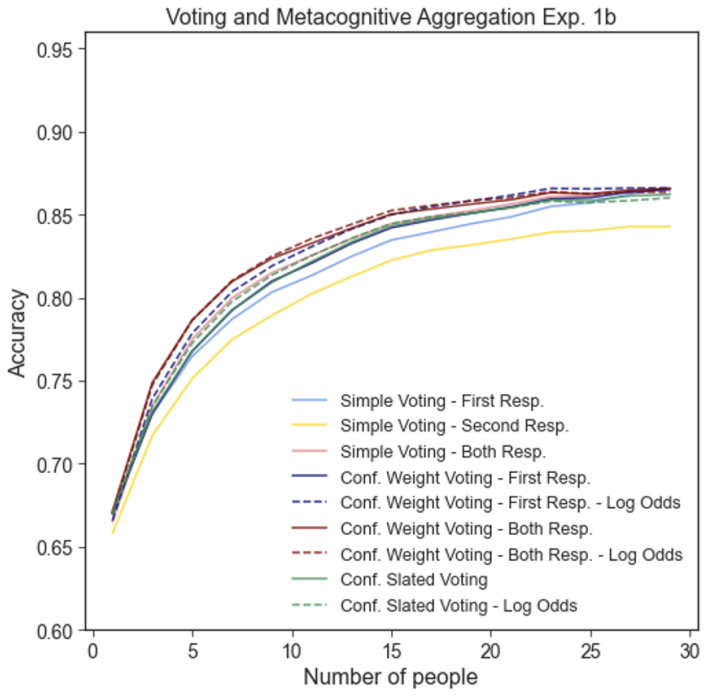

D

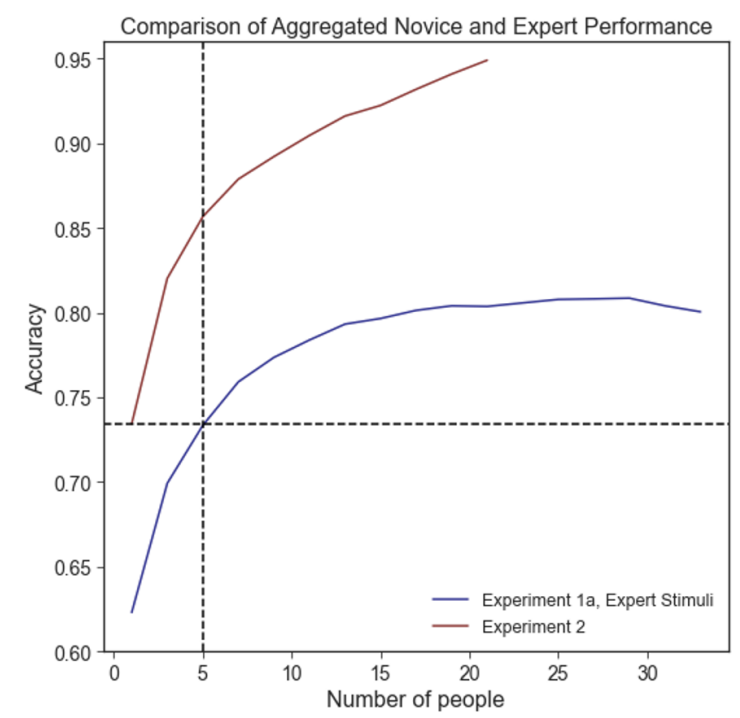

\section{Figure 4}

Panels A, B and C plot the accuracy obtained by applying all of our Wisdom of the Crowd algorithms (without normalization) to data from Experiments $1 \mathrm{a}, 1 \mathrm{~b}$, and 2 respectively. The legend for Panel B, C and D is identical to the one is Panel A. We observe that as the responses are pooled from more participants, the accuracy increases. Panel D compares the best performing algorithm, Confidence Weighted Voting with Both Responses, for novice participants from Experiment $1 \mathrm{a}$ and expert participants from Experiment 2. We restrict the responses from Experiment 1a to the stimuli that were used in the main trials of Experiment 2 and apply the algorithm. We observe that the performance of the expert participants is greater than that of the novice participants. We also observe that 5 novice participants can match the performance of 1 expert. 


\section{References}

Alialy, R., Tavakkol, S., Tavakkol, E., Ghorbani-Aghbologhi, A., Ghaffarieh, A., Kim, S. H., \& Shahabi, C. (2018). A review on the applications of crowdsourcing in human pathology. Journal of pathology informatics, 9.

Attali, Y., Budescu, D., \& Arieli-Attali, M. (2020). An item response approach to calibration of confidence judgments. Decision, 7(1), 1.

Bahrami, B., Olsen, K., Latham, P. E., Roepstorff, A., Rees, G., \& Frith, C. D. (2010). Optimally interacting minds. Science, 329(5995), 1081-1085.

Bang, D., Fusaroli, R., Tylén, K., Olsen, K., Latham, P. E., Lau, J. Y., Roepstorff, A., Rees, G., Frith, C. D., \& Bahrami, B. (2014). Does interaction matter? testing whether a confidence heuristic can replace interaction in collective decision-making. Consciousness and cognition, 26, 13-23.

Baron, J., Mellers, B. A., Tetlock, P. E., Stone, E., \& Ungar, L. H. (2014). Two reasons to make aggregated probability forecasts more extreme. Decision Analysis, 11(2), 133-145.

Baron, R. S. (2005). So right it's wrong: Groupthink and the ubiquitous nature of polarized group decision making.

Davis-Stober, C. P., Budescu, D. V., Dana, J., \& Broomell, S. B. (2014). When is a crowd wise? Decision, 1(2), 79.

Dissanayake, I., Nerur, S., Singh, R., \& Lee, Y. (2019). Medical crowdsourcing: Harnessing the "wisdom of the crowd" to solve medical mysteries. Journal of the Association for Information Systems, 20(11), 4.

Drew, T., Võ, M. L.-H., \& Wolfe, J. M. (2013). The invisible gorilla strikes again: Sustained inattentional blindness in expert observers. Psychological science, 24(9), 1848-1853.

Elmore, J. G., Nelson, H. D., Pepe, M. S., Longton, G. M., Tosteson, A. N., Geller, B., Onega, T., Carney, P. A., Jackson, S. L., Allison, K. H., \& Weaver, D. L. (2016). Variability in pathologists' interpretations of individual breast biopsy slides: A population perspective. Annals of internal medicine, 164(10), 649-655. 
Esser, J. K., \& Lindoerfer, J. S. (1989). Groupthink and the space shuttle challenger accident: Toward a quantitative case analysis. Journal of Behavioral Decision Making, 2(3), $167-177$.

Fleming, S. M., Dolan, R. J., \& Frith, C. D. (2012). Metacognition: Computation, biology and function.

Galton, F. (1907). Vox populi.

Griffin, D., \& Brenner, L. (2004). Perspectives on probability judgment calibration. Blackwell handbook of judgment and decision making, 177-199.

Griffin, D., \& Tversky, A. (1992). The weighing of evidence and the determinants of confidence. Cognitive psychology, 24(3), 411-435.

Hasan, E., Eichbaum, Q., Seegmiller, A. C., Stratton, C., \& Trueblood, J. S. (2021). Harnessing the wisdom of the confident crowd in medical image decision-making. Retrieved November 23, 2021, from osf.io/ckvxz

Herzog, S. M., \& Hertwig, R. (2009). The wisdom of many in one mind: Improving individual judgments with dialectical bootstrapping. Psychological Science, 20(2), 231-237.

Herzog, S. M., \& Hertwig, R. (2014). Harnessing the wisdom of the inner crowd. Trends in cognitive sciences, 18(10), 504-506.

Janis, I. L. (1973). Groupthink and group dynamics: A social psychological analysis of defective policy decisions. Policy Studies Journal, 2(1), 19.

Juni, M. Z., \& Eckstein, M. P. (2017). The wisdom of crowds for visual search. Proceedings of the National Academy of Sciences, 114(21), E4306-E4315.

Kamar, E., Hacker, S., \& Horvitz, E. (2012). Combining human and machine intelligence in large-scale crowdsourcing. AAMAS, 12, 467-474.

Koriat, A. (2012a). The self-consistency model of subjective confidence. Psychological review, 119(1), 80.

Koriat, A. (2012b). When are two heads better than one and why? Science, 336(6079), 360-362. 
Koriat, A. (2015). When two heads are better than one and when they can be worse: The amplification hypothesis. Journal of Experimental Psychology: General, 144(5), 934.

Kurvers, R. H., Herzog, S. M., Hertwig, R., Krause, J., Carney, P. A., Bogart, A., Argenziano, G., Zalaudek, I., \& Wolf, M. (2016). Boosting medical diagnostics by pooling independent judgments. Proceedings of the National Academy of Sciences, 113(31), 8777-8782.

Lee, M. D., \& Lee, M. N. (2017). The relationship between crowd majority and accuracy for binary decisions. Judgment and Decision Making, 12(4), 328.

Liberman, V., \& Tversky, A. (1993). On the evaluation of probability judgments: Calibration, resolution, and monotonicity. Psychological Bulletin, 114(1), 162.

Litvinova, A., Herzog, S. M., Kall, A. A., Pleskac, T. J., \& Hertwig, R. (2020). How the "wisdom of the inner crowd" can boost accuracy of confidence judgments. Decision, 7(3), 183.

Litvinova, A., Kurvers, R. H., Hertwig, R., \& Herzog, S. M. (2022). How experts’ own inconsistency relates to their confidence and between-expert disagreement. Scientific Reports, 12(1), 1-12.

Meyen, S., Sigg, D. M., von Luxburg, U., \& Franz, V. H. (2021). Group decisions based on confidence weighted majority voting. Cognitive research: principles and implications, 6(1), 1-13.

Montgomery, L. E., \& Lee, M. (2022). The wisdom of the crowd and framing effects in spatial knowledge. Proceedings of the Annual Meeting of the Cognitive Science Society, 44(44).

Mozafari, B., Sarkar, P., Franklin, M., Jordan, M., \& Madden, S. (2014). Scaling up crowd-sourcing to very large datasets: A case for active learning. Proceedings of the VLDB Endowment, 8(2), 125-136.

Nelson, A. M., Hale, M., Diomande, M. I. J.-M., Eichbaum, Q., Iliyasu, Y., Kalengayi, R. M., Rugwizangoga, B., \& Sayed, S. (2018). Training the next generation of african pathologists. Clinics in Laboratory Medicine, 38(1), 37-51.

Olsson, H. (2014). Measuring overconfidence: Methodological problems and statistical artifacts. Journal of Business Research, 67(8), 1766-1770. 
Ørting, S. N., Doyle, A., van Hilten, A., Hirth, M., Inel, O., Madan, C. R., Mavridis, P., Spiers, H., \& Cheplygina, V. (2020). A survey of crowdsourcing in medical image analysis. Human Computation, 7(1), 1-26.

Pallier, G., Wilkinson, R., Danthiir, V., Kleitman, S., Knezevic, G., Stankov, L., \& Roberts, R. D. (2002). The role of individual differences in the accuracy of confidence judgments. The Journal of general psychology, 129(3), 257-299.

Press, G. (2021). Centaur labs gets $\$ 15$ million to improve data for healthcare ai. Forbes. https://www.forbes.com/sites/gilpress/2021/09/03/centaur-labs-gets-15-million-toimprove-data-for-healthcare-ai/?sh=139e900763a0

Rahnev, D., \& Fleming, S. M. (2019). How experimental procedures influence estimates of metacognitive ability. Neuroscience of consciousness, 2019(1), niz009.

Saposnik, G., Redelmeier, D., Ruff, C. C., \& Tobler, P. N. (2016). Cognitive biases associated with medical decisions: A systematic review. BMC medical informatics and decision making, 16(1), 1-14.

Shekhar, M., \& Rahnev, D. (2021). Sources of metacognitive inefficiency. Trends in Cognitive Sciences, 25(1), 12-23.

Steyvers, M., Wallsten, T. S., Merkle, E. C., \& Turner, B. M. (2014). Evaluating probabilistic forecasts with bayesian signal detection models. Risk Analysis, 34(3), 435-452.

Stroop, J. R. (1932). Is the judgment of the group better than that of the average member of the group? Journal of experimental Psychology, 15(5), 550.

Surowiecki, J. (2005). The wisdom of crowds. Anchor.

Trueblood, J. S., Eichbaum, Q., Seegmiller, A. C., Stratton, C., O’Daniels, P., \& Holmes, W. R. (2021). Disentangling prevalence induced biases in medical image decision-making. Cognition, 212, 104713.

Trueblood, J. S., Holmes, W. R., Seegmiller, A. C., Douds, J., Compton, M., Szentirmai, E., Woodruff, M., Huang, W., Stratton, C., \& Eichbaum, Q. (2018). The impact of speed and 
bias on the cognitive processes of experts and novices in medical image decision-making. Cognitive Research: Principles and Implications, 3(1), 1-14.

Tullis, J. G., \& Goldstone, R. L. (2020). Why does peer instruction benefit student learning? Cognitive research: principles and implications, 5(1), 1-12.

Turner, B. M., Steyvers, M., Merkle, E. C., Budescu, D. V., \& Wallsten, T. S. (2014). Forecast aggregation via recalibration. Machine learning, 95(3), 261-289.

Vul, E., \& Pashler, H. (2008). Measuring the crowd within: Probabilistic representations within individuals. Psychological Science, 19(7), 645-647.

Waite, S., Scott, J., Gale, B., Fuchs, T., Kolla, S., \& Reede, D. (2017). Interpretive error in radiology. American Journal of Roentgenology, 208(4), 739-749.

Wolf, M., Krause, J., Carney, P. A., Bogart, A., \& Kurvers, R. H. (2015). Collective intelligence meets medical decision-making: The collective outperforms the best radiologist. PloS one, 10(8), e0134269.

Yates, J. F. (1982). External correspondence: Decompositions of the mean probability score. Organizational Behavior and Human Performance, 30(1), 132-156.

Yeung, N., \& Summerfield, C. (2012). Metacognition in human decision-making: Confidence and error monitoring. Philosophical Transactions of the Royal Society B: Biological Sciences, 367(1594), 1310-1321. 